\title{
Challenges and obstacles for a bouncing universe in brane models
}

\author{
P. Kanti and K. Tamvakis* \\ CERN, Geneva, Switzerland \\ (Received 18 March 2003; published 10 July 2003)
}

\begin{abstract}
A brane evolving in the background of a charged AdS black hole displays in general a bouncing behavior with a smooth transition from a contracting to an expanding phase. We examine in detail the conditions and consequences of this behavior in various cases. For a cosmological-constant-dominated brane, we obtain a singularity-free, inflationary era which is shown to be compatible only with an intermediate-scale fundamental Planck mass. For a radiation-dominated brane, the bouncing behavior can occur only for background-charge values exceeding those allowed for non-extremal black holes. For a matter-dominated brane, the black-hole mass affects the proper volume or the expansion rate of the brane. We also consider the brane evolving in an asymmetric background of two distinct charged AdS black hole spacetimes being bounded by the brane and find that, in the case of an empty critical brane, bouncing behavior occurs only if the black-hole mass difference is smaller than a certain value. The effects of a brane curvature term on the bounce at early and late times are also investigated.
\end{abstract}

DOI: 10.1103/PhysRevD.68.024014

PACS number(s): 04.50.+h, 98.80.Cq

\section{INTRODUCTION}

The idea of realizing our Universe as a defect [1] in a higher-dimensional spacetime has received a lot of attention in the recent years after the introduction of D-branes [2], i.e. membranes on which the fundamental string fields satisfy Dirichlet boundary conditions. Motivated by string or $\mathrm{M}$ theory [3] and the AdS/Conformal Field Theory (CFT) correspondence [4], brane models have revealed new possibilities for the resolution of the hierarchy problem of particle physics [5-8]. The D-brane is assigned an intrinsic energy density and pressure arising both from an underlying brane tension and from ordinary $(3+1)$-dimensional matter trapped on it by stringy effects. Gravitons, on the other hand, propagate into the higher-dimensional bulk. Nevertheless, as it turns out in the Randall-Sundrum model (RS) [9,10], virtual gravitons are localized near the brane due to the curvature of the higher-dimensional bulk. In this model, our spacetime is embedded in a higher dimensional space with an extra highly curved but infinite fifth dimension. The localization distance of gravity is proportional to the characteristic length defined by the cosmological constant of the anti-de Sitter (AdS) bulk space. While the Poincare-invariant RS solution requires a fine-tuning of the brane tension, nonPoincaré-invariant solutions are also possible. A fourdimensional Friedmann-Robertson-Walker (FRW) universe can arise on a brane embedded in an AdS bulk [11] or an AdS-black-hole bulk spacetime $[12,13]$. However, in both cases, the presence of extra terms, remnants from higherdimensional theory, may lead to modifications in the evolution of the brane at small scales.

The brane-world framework that will be followed in this paper consists of our physical universe being regarded as a $(3+1)$-dimensional hypersurface embedded in a $(4+1)$ dimensional AdS bulk. The recent observational evidence of cosmological acceleration motivates the consideration not

\footnotetext{
*On leave from the University of Ioannina, Greece.
}

only of a critical brane of a vanishing four-dimensional cosmological constant but also of a non-critical brane as well. The bulk space background will be taken to be that of a (4 +1)-dimensional AdS black hole $[12,14,15]$ with charge [16]. Recent investigations $[17,18]$ seem to indicate that, due to the non-vanishing charge, a bouncing universe could, in principle, arise, i.e. a universe that bounces from a contracting phase to an expanding one without encountering a singularity (see also [13] and [19-28]; for earlier examples of singularity-free solutions in the framework of superstring theory, see [29]). Reference [17] considers a semi-realistic radiation-dominated brane, while Ref. [18] studies a generally non-critical but empty brane.

In the present article, we extend existing studies considering a brane evolving in a charged AdS black hole background. After presenting the theoretical framework of our analysis and briefly reviewing the derivation of the Friedmann equation on the brane, we reconsider the evolution of both a critical and non-critical brane. In the former case, we reconfirm the occurrence of a bounce at small scales that renders the solutions free from both past and future singularities. In the latter case, the singularity-free, early regime is followed by an asymptotically expanding de Sitter epoch, the sequence of which successfully models an early, inflationary period. We find that the asymptotic Hubble parameter for the expansion on the brane is bounded from above by the blackhole mass and that this model is compatible only with intermediate-scale gravitational theories, i.e. with $M_{5}$ $>10^{-5} M_{P}$. We then proceed to study the evolution of a radiation-dominated brane and to derive the exact solution for the scale factor of the four-dimensional subspace, which is indeed characterized by a non-vanishing minimum value. A careful examination, however, reveals that the bouncing behavior for a radiation-dominated brane occurs for background-charge values exceeding those allowed for nonextremal black holes. Finally, in the case of a brane filled with a matter energy density, the presence of the charge bulk parameter ensures once again the avoidance of the future singularity in the case of a closed universe. The presence of 
the black-hole mass, which survives in the Friedmann equation at large scales during the same period, also affects the evolution on the brane: the main implication for a closed universe is the increase in its proper volume while, for a flat and open universe, this term increases or decreases, respectively, the rate of expansion.

We then proceed to consider a brane evolving in an asymmetric background of two distinct charged AdS black-hole spacetimes being bounded by the brane. In the case of an empty critical brane, we find that, for the occurrence of a bounce, the asymmetry in the black-hole mass parameters has to be smaller than a certain value, in contrast to the symmetric case where bouncing behavior occurs generically. For a radiation- or matter-dominated brane, the effect of the asymmetry is irrelevant both at small and large scales. We finally assume the presence of an intrinsic boundary curvature term in the action which is expected to modify the evolution of the universe only at large scales. We show that, indeed, the value of the scale factor at the bouncing point does not depend on this term, even for large values of the associated parameter that determines the magnitude of this term in the action, and that the only effects coming from the boundary curvature term are relevant in the large scale factor regime.

\section{THE (4+1)-DIMENSIONAL CHARGED BLACK HOLE BACKGROUND}

We shall consider the following $(4+1)$-dimensional gravitational theory described by the action

$$
\begin{aligned}
\mathcal{S}= & \frac{1}{16 \pi G} \int_{M} d^{5} x \sqrt{-g}\left(\mathcal{R}_{5}+\frac{12}{\ell^{2}}-F_{M N} F^{M N}\right) \\
& +\frac{1}{8 \pi G} \int_{\partial M} d^{4} x \sqrt{-\gamma} \mathcal{K},
\end{aligned}
$$

where $\mathcal{R}_{5}$ denotes the scalar curvature of the 5-dimensional spacetime, $\ell$ is the AdS curvature length related to the bulk cosmological constant through $\Lambda_{5}=-6 / \ell^{2}$, and $F_{M N}$ stands for the field strength of a bulk gauge field. The bulk space $M$ consists in general of two different regions separated by the hypersurface $\partial M$ signifying the brane, the simplest choice being two regions related by a $\mathcal{Z}_{2}$ symmetry. $\mathcal{K}$ is the trace of the extrinsic curvature on $\partial M$ defined as $\mathcal{K}_{M N}=\nabla_{M} \eta_{N}$ in terms of the unit normal on it. Finally, $\gamma_{\mu \nu}$ is the induced metric on the boundary and $G$ the five-dimensional Newton constant.

In addition to the above, we assume a term $\int d^{4} x \sqrt{-\gamma} \mathcal{L}$ from which a conserved four-dimensional energy-momentum tensor $T_{\mu \nu}$ arises, satisfying the Israel junction conditions

$$
\Delta \mathcal{K}_{\mu \nu} \equiv \mathcal{K}_{\mu \nu}^{(+)}-\mathcal{K}_{\mu \nu}^{(-)}=-8 \pi G\left(T_{\mu \nu}-\frac{1}{3} T_{\lambda}^{\lambda} \gamma_{\mu \nu}\right)
$$

Einstein's equations in the bulk are satisfied by the $A d S$ charged black hole background metric

$$
d s_{5}^{2}=-f(r) d t^{2}+f^{-1}(r) d r^{2}+r^{2} d \Omega_{3, k}^{2}
$$

with

$$
f(r) \equiv \frac{r^{2}}{\ell^{2}}+k-\frac{\mu}{r^{2}}+\frac{q^{2}}{r^{4}} .
$$

In the above, $d \Omega_{3, k}^{2}$ stands for a 3D spatial geometry with the topology of a plane $(k=0)$, a sphere $(k=1)$ or a hyperboloid $(k=-1)$. The parameters appearing in the metric function $f(r)$ are related to the Arnowitt-Deser-Misner (ADM) mass and charge parameters of the black hole through ${ }^{1}$

$$
\mu \equiv \omega_{4} M, \quad q^{2} \equiv 3 \omega_{4}^{2} Q^{2} / 16,
$$

with $\omega_{4}=16 \pi G / 3 \Omega_{3}=8 G / 3 \pi$.

The black hole possesses in general two horizons the position of which is determined by the solution of the cubic equation $f(r)=0$. It will be useful, at this point, to introduce the dimensionless parameters and variables $\bar{\mu} \equiv \mu / \ell^{2}, \bar{q}^{2}$ $\equiv q^{2} / \ell^{4}$ and $y \equiv r^{2} / \ell^{2}$. Then, the case of two distinct horizons corresponds to values of the charge

$$
\bar{q}^{2}<\bar{q}_{+}^{2},
$$

where

$$
\bar{q}_{ \pm}^{2} \equiv-\frac{k}{3}\left(\bar{\mu}+\frac{2}{9} k^{2}\right) \pm \frac{2}{3 \sqrt{3}}\left(\bar{\mu}+\frac{k^{2}}{3}\right)^{3 / 2} .
$$

Note that always $\bar{q}_{+}^{2}>0$ and $\bar{q}_{-}^{2}<0$. The two horizons correspond to the two positive solutions of a cubic equation, namely

$$
\begin{gathered}
y_{\text {out }}=-\frac{k}{3}+2\left(\frac{\bar{\mu}}{3}+\frac{k^{2}}{9}\right)^{1 / 2} \cos (\phi-\pi / 3) \\
y_{\text {in }}=-\frac{k}{3}+2\left(\frac{\bar{\mu}}{3}+\frac{k^{2}}{9}\right)^{1 / 2} \sin (\pi / 6-\phi),
\end{gathered}
$$

where we have introduced

\footnotetext{
${ }^{1}$ The charge $Q$ is associated with an Abelian gauge field defined in the bulk and has nothing to do with the usual electric charge carried by brane matter. All standard model fields are assumed to be strictly localized on the brane. The two black-hole bulk spacetimes, whose common boundary is the 3-brane, are characterized by charges of equal value but opposite signs, an assumption which is consistent with the $\mathcal{Z}_{2}$-symmetry. In this way, the lines of the Abelian bulk field start from the positive charge and end at the negative one, extending continuously over the brane, and the Abelian flux is conserved without having to introduce additional charges on the brane. By using the AdS/CFT correspondence, one can derive the form of the potential at the location of the brane $[17,27,30,31]$ which reads $\Phi=\ell \phi / r$, where $\phi$ is the 5-dimensional potential given by $\phi$ $=3 \omega_{4} Q / 8 r^{2}$.
} 


$$
\phi \equiv \frac{1}{3} \tan ^{-1}\left(\frac{2}{\sqrt{\frac{q^{2}-q_{-}^{2}}{q_{+}^{2}-q^{2}}}-\sqrt{\frac{q_{+}^{2}-q^{2}}{q^{2}-q_{-}^{2}}}}\right) .
$$

For values of the charge larger than the limiting charge, namely for $q^{2}>q_{+}^{2}$, two of the roots of the horizon equation are complex and there is only one horizon. Thus, in this case we have an extremal black hole. The stability status of extremal black holes is still an open question [31] and, perhaps, they should be avoided as a background.

\section{BRANE WORLD IN A CHARGED BLACK HOLE BACKGROUND}

Following the steps of Ref. [12] and introducing a spherically symmetric 3-brane at the position $r=R$, we obtain from the Israel junction conditions the following Friedmann equation on the brane:

$$
\frac{\left[f(R)+\dot{R}^{2}\right]^{1 / 2}}{R}=\frac{4 \pi G}{3}(\rho+\sigma),
$$

where the overdot denotes the derivative with respect to the proper time $\tau$ on the brane. In addition, $\rho$ is the matter energy density on the brane and $\sigma$ the brane tension. The last two arise from the brane energy-momentum tensor $T_{0}^{0}=$ $-(\rho+\sigma), T_{j}^{i}=\delta_{j}^{i} p$, conserved through the equation

$$
\nabla_{\mu} T_{\nu}^{\mu}=0 \Rightarrow \dot{\rho}+3 \frac{\dot{R}}{R}(\rho+p)=0 .
$$

Note that this equation is derived only in the case of $\mathcal{Z}_{2}$ symmetry. ${ }^{2}$ In an asymmetric situation, we have the more general equation

$$
\frac{\left[f_{+}(R)+\dot{R}^{2}\right]^{1 / 2}}{R}+\frac{\left[f_{-}(R)+\dot{R}^{2}\right]^{1 / 2}}{R}=\frac{8 \pi G}{3}(\rho+\sigma) .
$$

The metric functions $f_{ \pm}(r)$ can differ in the vacuum parameters $\mu_{ \pm}$and $\ell_{ \pm}$.

The four-dimensional metric on the brane corresponds to a FRW universe, $R(\tau)$ being the scale factor. It is

$$
d s_{4}^{2}=-d \tau^{2}+R^{2}(\tau) d \Omega_{3, k}^{2} .
$$

Taking the square of the Friedmann equation (11), we obtain the more conventional form

$$
\begin{aligned}
H^{2} \equiv & \left(\frac{\dot{R}}{R}\right)^{2}=-\frac{k}{R^{2}}+\frac{\mu}{R^{4}}-\frac{q^{2}}{R^{6}}+\frac{8 \pi G_{4}}{3} \Lambda_{4} \\
& +\left(\frac{4 \pi G}{3}\right)^{2}\left(\rho^{2}+2 \sigma \rho\right) .
\end{aligned}
$$

\footnotetext{
${ }^{2}$ The radius $r$ is decreasing on both sides of the brane.
}

In the above, we have defined the four-dimensional cosmological constant as $^{3}$

$$
\frac{8 \pi G_{4}}{3} \Lambda_{4} \equiv\left(\frac{4 \pi G \sigma}{3}\right)^{2}-\frac{1}{\ell^{2}} \geqslant 0
$$

The generic case is that of a de Sitter brane. The case $\Lambda_{4}$ $=0$ of a critical brane is achieved through the well-known fine-tuning between bulk $(G, \ell)$ and brane $(\sigma)$ parameters of the Randall-Sundrum model.

The above Friedmann equation of the brane features a dark energy term $\mu / R^{4}$ that has the same scale dependence as the standard radiation term. ${ }^{4}$ The term arising from the presence of the bulk charge corresponds to a stiff energy equation of state $(w=1)$ characterized by an exotic negative energy density.

\section{REVIEW OF THE EVOLUTION OF AN EMPTY BRANE}

It is instructive to review, and complete, the solutions of the Friedmann equation in the case of an empty brane $[14,15,17,18]$ before proceeding to study more realistic cases. For $\rho=0$, Eq. (15) takes the form

$$
\left(\frac{\dot{R}}{R}\right)^{2}=-\frac{k}{R^{2}}+\frac{\mu}{R^{4}}-\frac{q^{2}}{R^{6}}+\frac{8 \pi G_{4}}{3} \Lambda_{4} .
$$

We will now consider separately the cases of a critical $\left(\Lambda_{4}=0\right)$ and non-critical $\left(\Lambda_{4} \neq 0\right)$ brane.

\section{A. Empty, critical brane}

By assuming an empty and critical brane $\left(\Lambda_{4}=0\right)$ and introducing a new time variable $d \tau=R(\eta) d \eta$, Eq. (17) leads to the solutions shown in Table I, for $k=0, \pm 1$. The parameter $\epsilon$ is defined as $\epsilon^{2}=4 q^{2} / \mu^{2}$. All three solutions are characterized by a minimum radius of contraction beyond which the universe bounces to an expanding phase. Thus, there is no primordial or future singularity associated with these cosmologies. Note that the radius at which the bouncing occurs is always outside of the outer horizon of the black hole, since $H^{2}=-f(R) / R^{2}+(8 \pi G / 3) \sigma^{2}=0$ implies $f(R)$ $=(8 \pi G / 3) \sigma^{2} R^{2}>0$.

The solution for $k=1$ is periodic and is characterized by an infinite number of bounces at the two points $R_{\min }$ and $R_{\max }$. This solution does not possess either a big bang or big crunch singularity, and it is possible only for $\epsilon^{2}<1$. This restriction on the black hole charge $\left(q^{2}<\mu^{2} / 4\right)$ is always satisfied if the black hole of the background has two horizons. Indeed, recalling the corresponding constraint $\bar{q}^{2}$

\footnotetext{
${ }^{3}$ The four-dimensional Newton constant can be read off from the linear energy-density term to be $8 \pi G_{4} / 3 \equiv 2 \sigma(4 \pi G / 3)^{2}$.

${ }^{4}$ The equation of state $p=w \rho$, through the conservation equation $\dot{\rho} / \rho=-3(1+w) \dot{R} / R$, in the case of radiation $(w=-1 / 3)$, corresponds to $\rho \propto R^{-4}$.
} 
TABLE I. Charged $\left(Q^{2} \neq 0\right)$, critical $\left(\Lambda_{4}=0\right)$ brane.

\begin{tabular}{|c|c|c|c|}
\hline$k=1$ & $R^{2}=\frac{\mu}{2}\left(1-\sqrt{1-\epsilon^{2}} \cos 2 \eta\right)$ & $\begin{array}{c}R_{\min }^{2}=\frac{\mu}{2}\left(1-\sqrt{1-\epsilon^{2}}\right) \\
R_{\max }^{2}=\mu / 2, \epsilon^{2}<1\end{array}$ & $\begin{array}{l}\text { Bouncing } \\
\text { cyclic }\end{array}$ \\
\hline$k=0$ & $R^{2}=\frac{\mu}{4}\left(\epsilon^{2}+4 \eta^{2}\right)$ & $R_{\min }^{2}=\mu \epsilon^{2} / 4, R_{\max }^{2}=\infty$ & $\begin{array}{l}\text { Bouncing } \\
\text { expanding }\end{array}$ \\
\hline$k=-1$ & $R^{2}=\frac{\mu}{2}\left(-1+\sqrt{1+\epsilon^{2}} \cosh 2 \eta\right)$ & $\begin{aligned} R_{\min }^{2}= & \frac{\mu}{2}\left(\sqrt{1+\epsilon^{2}}-1\right) \\
& R_{\max }^{2}=\infty\end{aligned}$ & $\begin{array}{l}\text { Bouncing } \\
\text { expanding }\end{array}$ \\
\hline
\end{tabular}

$<\bar{q}_{+}^{2}$, we see that $\bar{q}_{+}^{2}$ is always smaller than $\bar{\mu}^{2} / 4$. A simple numerical analysis shows that $\bar{\mu}^{2} / 4+\frac{1}{3}\left(\bar{\mu}+\frac{2}{9}\right)-(2 / 3 \sqrt{3})(\bar{\mu}$ $\left.+\frac{1}{3}\right)^{3 / 2}$ is always positive for any value $0<\bar{\mu}<\infty$. By using dimensionful parameters, we may write the allowed range of values of the added black hole charge, for the existence of physically acceptable bouncing universes in a two-horizon black hole background, as

$$
0<Q^{2}<\frac{16}{3 \omega_{4}^{2}}\left[-\frac{\ell^{2}}{3}\left(\omega_{4} M+\frac{2 \ell^{2}}{9}\right)+\frac{2 \ell}{3 \sqrt{3}}\left(\omega_{4} M+\frac{\ell^{2}}{3}\right)^{3 / 2}\right]
$$

for a given ADM black hole mass $M$ and AdS curvature length $\ell$. Alternatively, for fixed $M$ and $Q^{2}$, the above constraint may be interpreted as a lower bound on the AdS curvature $\ell$ or, through the relation $\sigma=3 / 4 \pi G \ell$ for a critical brane, as an upper bound on the tension $\sigma$ of such a brane, which is introduced in the aforementioned background.

For $k=0,-1$, the solutions are characterized by a single bounce that demands again a non-vanishing value of the black hole charge. The constraint for the existence of two horizons still needs to be satisfied and reduces to

$$
Q^{2}<\frac{32 M^{2} \ell}{9 \sqrt{3 \omega_{4} M}}
$$

for $k=0$, and to Eq. (18) with the sign of the first term on the right-hand side reversed, for $k=-1$.

\section{B. Empty, non-critical brane}

In the case of non-vanishing four-dimensional cosmological constant $\Lambda_{4}$, the Friedmann equation is modified only for very large values of the scale factor $R$. The short distance behavior is dominated by the mass and charge terms. Thus, the $\Lambda_{4} \neq 0$ solutions at short distances are very close to the previously discussed set, while for large distances they are very close to the solutions of the Friedmann equation with a vanishing charge, since at those distances the charge term becomes irrelevant. The latter set are given in Table II, where we have defined a new parameter $\bar{\epsilon}$ through the relation $\bar{\epsilon}^{2}$ $\equiv 4 \kappa_{4}^{2} \mu \Lambda_{4} / 3$, with $\kappa_{4}^{2}=8 \pi G_{4}$.

Joining together the two sets of solutions presented in these two subsections, we see that the cosmology of an empty brane with a non-vanishing cosmological constant possesses a bouncing point at early times and can have a generic expanding behavior at late times. This can be a plausible scenario for an early inflationary era in which the cosmological constant stands for, or includes, an almost constant energy density of a scalar (inflaton) field. The bounce at early times guarantees the absence of a big bang singularity ${ }^{5}$ for all values of $k$ as long as the bounds on the charge parameter $Q$ presented in the previous subsection are respected. At late times, an additional constraint arises, for $k= \pm 1$, for the validity of the solutions, namely $\bar{\epsilon}^{2}<1$. This constraint leads to an upper bound on the Hubble parameter of the asymptotic expansion on the brane in terms of bulk parameters, namely

$$
H_{\infty}^{2} \equiv \frac{\kappa_{4}^{2}}{3} \Lambda_{4}<\frac{1}{4 \omega_{4} M}=\frac{3 \pi M_{5}^{3}}{32 M},
$$

where $M_{5}$ is the fundamental scale of gravity in five dimensions. If this period of asymptotic exponential expansion plays the role of standard inflation, then the vacuum energy density of the brane must be of order $\Lambda_{4} \sim\left(10^{16} \mathrm{GeV}\right)^{4}$ in order to obtain the correct magnitude of density perturbations. This, in conjunction to Eq. (20), leads to

$$
\left(\frac{M_{5}}{M_{P}}\right)^{2}>10^{-11}\left(\frac{M}{M_{5}}\right) \text {. }
$$

Assuming that the black hole mass is at least $M \geqslant 10 M_{5}$, the above constraint puts a lower bound on the value of the five-dimensional Planck scale, i.e. $M_{5}>10^{-5} M_{P}$, in agreement with similar bounds found in the literature for the occurrence of brane inflation in higher-dimensional models [32]. Alternatively, pushing the scale of gravity down to the $\mathrm{TeV}$ scale leads to a black hole mass which is many orders of magnitude below the fundamental scale, a result that invalidates the classical field theory approach used in our analysis.

\section{RADIATION DOMINATED BRANE}

Let us now consider the realistic case of a brane with a non-zero energy density that obeys a radiation equation of state $(w=1 / 3)$ and has a scale factor dependence of the form

\footnotetext{
${ }^{5}$ Note, however, that the periodic behavior of the critical $k=1$ case that describes a cyclic universe is not retained in the presence of a cosmological constant.
} 
TABLE II. Neutral $\left(Q^{2}=0\right)$, non-critical $\left(\Lambda_{4} \neq 0\right)$ brane.

\begin{tabular}{|c|c|c|c|}
\hline$k=1$ & $R^{2}=\frac{3}{2 \kappa_{4}^{2} \Lambda_{4}} \mid 1+\sqrt{1-\bar{\epsilon}^{2}} \cosh \left(2 \kappa_{4} \sqrt{\frac{\Lambda_{4}}{3}\left(\tau-\tau_{0}\right)}\right)$ & $R_{\infty}^{2} \propto e^{2 \kappa_{4}\left(\sqrt{\Lambda_{4} / 3}\right) \tau}$ & asymptotic dS \\
\hline$k=0$ & $R^{2}=\sqrt{\frac{3 \mu}{\kappa_{4}^{2} \Lambda_{4}}} \sinh \left(2 \kappa_{4} \sqrt{\frac{\Lambda_{4}}{3}}\left(\tau-\tau_{0}\right)\right)$ & $R_{\infty}^{2} \propto e^{2 \kappa_{4}\left(\sqrt{\Lambda_{4} / 3}\right) \tau}$ & asymptotic dS \\
\hline$k=-1$ & $R^{2}=\frac{3}{2 \kappa_{4}^{2} \Lambda_{4}}\left[-1+\sqrt{1-\bar{\epsilon}^{2}} \cosh \left(2 \kappa_{4} \sqrt{\frac{\Lambda_{4}}{3}}\left(\tau-\tau_{0}\right)\right.\right.$ & $R_{\infty}^{2} \propto e^{2 \kappa_{4}\left(\sqrt{\Lambda_{4} / 3}\right) \tau}$ & asymptotic dS \\
\hline
\end{tabular}

$\rho=\hat{\rho} / R^{4}$. Going back to the Friedmann equation (15) and substituting the energy density, we obtain

$$
\begin{aligned}
H^{2}= & \left(\frac{\dot{R}}{R}\right)^{2}=\frac{8 \pi G_{4}}{3} \Lambda_{4}-\frac{k}{R^{2}}+\left(\mu+\frac{8 \pi G_{4}}{3} \hat{\rho}\right) \frac{1}{R^{4}}-\frac{q^{2}}{R^{6}} \\
& +\frac{4 \pi G_{4}}{3 \sigma} \frac{\hat{\rho}^{2}}{R^{8}} .
\end{aligned}
$$

In what follows, we solve the above equation both for early and late times and proceed to check the validity of the derived bouncing solution.

\section{A. Derivation of the solution}

There are two distinct scale regimes at which different terms dominate. For small scale factors, we may neglect the cosmological constant $\Lambda_{4}$ and the curvature term $k / R^{2}$. This is the early regime that should be responsible for the existence of a bounce and the avoidance of the primordial singularity. Introducing the new variables $x \equiv R^{2}$ and $d \tau$ $=R^{2} d \bar{\tau}$, the approximate Friedmann equation can take the form

$$
\frac{1}{4}\left(x^{\prime}\right)^{2}=b x^{2}-q^{2} x+a
$$

where we have defined

$$
a \equiv \frac{4 \pi G_{4} \hat{\rho}^{2}}{3 \sigma}, \quad b \equiv \mu+\frac{8 \pi G_{4}}{3} \hat{\rho}=(1+\lambda) \frac{8 \pi G_{4}}{3} \hat{\rho}
$$

and the prime denotes differentiation with respect to the new time $\bar{\tau}$. We have also introduced, for later use, a new dimensionless parameter $\lambda$ defined through the relation $\mu$ $=\lambda\left[\left(8 \pi G_{4} / 3\right) \hat{\rho}\right]$. By setting $x^{\prime}=0$ and demanding the existence of a bounce, the following condition on the minimum value of the charge parameter emerges:

$$
q^{4} \geqslant 4 a b=\frac{2 \hat{\rho}}{\sigma}(1+\lambda)\left(\frac{8 \pi G_{4}}{3} \hat{\rho}\right)^{2}
$$

On the other hand, the solution of Eq. (23) has the form

$$
R^{2}=\frac{q^{2}}{2 b}+\sqrt{\Delta} \cosh (2 \sqrt{b} \bar{\tau})
$$

where $\Delta=-a / b+\left(q^{2} / 2 b\right)^{2}>0$. The time variables are related through

$$
\tau=\frac{q^{2}}{2 b} \bar{\tau}+\sqrt{\frac{\Delta}{4 b}} \sinh (2 \sqrt{b} \bar{\tau})
$$

It is clear that there is a non-zero minimum value of the scale factor

$$
R_{\min }^{2}=\frac{q^{2}}{2 b}+\sqrt{\Delta}
$$

where the bounce occurs [an arbitrary integration constant in Eq. (26) has been chosen such that the point $\bar{\tau}=0$ coincides with the time of the bouncing].

For large scale factors, the charge term, and the quadratic energy-density term, due to their scale factor dependence, are suppressed and thus can be neglected. The resulting Friedmann equation for this late regime is

$$
H^{2} \simeq \frac{8 \pi G_{4}}{3} \Lambda_{4}-\frac{k}{R^{2}}+\frac{b}{R^{4}}
$$

and coincides in form with the one for a non-critical empty brane. The corresponding solutions therefore can be obtained from Table II of Sec. IV with the replacement $\mu \rightarrow b$ and $\bar{\epsilon}^{2} \rightarrow 4 b \kappa_{4}^{2} \Lambda_{4} / 3$. They all describe an asymptotically de Sitter expanding universe. Combining the derived early and late time solutions, we can successfully model an early, singularity-free, radiation-dominated epoch that passes smoothly to an inflationary period for the universe. In this case, the constraint $\bar{\epsilon}^{2}<1$ puts an upper bound on the asymptotic Hubble parameter during inflation, i.e.

$$
H_{\infty}^{2} \equiv \frac{\kappa_{4}^{2}}{3} \Lambda_{4}<\frac{1}{4}\left(\omega_{4} M+\frac{8 \pi}{3} \frac{\hat{\rho}}{M_{P}^{2}}\right)^{-1},
$$

in terms of the black hole mass as well as the energy density of the precedented radiation-dominated epoch. Viewing the above inequality as a constraint on the ratio between the five and four-dimensional Planck scales, we obtain a bound which is even stricter than the one derived in the case of an 
empty, non-critical brane. For negligible values of the parameter $\hat{\rho}$, we recover Eq. (21) and the constraint for intermediate gravity scale, while for large values of $\hat{\rho}, M_{5}$ is pushed further towards $M_{P}$.

In the case of a vanishing four-dimensional cosmological constant (or sub-dominant compared to the linear energydensity term), we recover at late times a standard, radiationtype Friedmann equation that describes a radiationdominated epoch well after the end of inflation. The solutions in that late regime, for all values of $k$, can be obtained from the ones presented in Table I of Sec. IV by setting $Q^{2}=0$ and $\mu \rightarrow b$. These solutions should duplicate exactly the successful cosmological predictions for nucleosynthesis. As has been noted in the literature before [33$35,17]$, this puts a strong bound on any non-standard contribution to the energy density, and thus on the black hole mass parameter, that has the same scaling as the linear radiation term. The dark radiation term generated by it should not exceed the effect that an additional neutrino species would have on the value of the $R^{-4}$ coefficient. This amounts to $\mu<1.13 G_{4} \hat{\rho}$ or, equivalently, to $\lambda<0.14$.

\section{B. Validity of the Bouncing Solution}

The occurrence of a bounce in a radiation-dominated brane requires, as we saw, a bulk charge larger than a minimum value that depends on the radiation energy density, namely

$$
q^{4} \geqslant \frac{2 \hat{\rho}}{\sigma}(1+\lambda)\left(\frac{8 \pi G_{4}}{3} \hat{\rho}\right)^{2}
$$

Nevertheless, as we discussed in Sec. II, the charge of the bulk background metric cannot increase further than a limiting value $q_{+}^{2}$ determined by the mass of the black hole, since beyond that charge the two horizons merge giving us an extremal black hole the stability of which is questionable. Since neither the curvature $k$ nor the cosmological constant are of importance in the regime where the bounce occurs, it is sufficient to consider this bound in the critical and flat case. It is

$$
q^{2}<q_{+}^{2}=\ell^{4} \frac{2}{3 \sqrt{3}} \bar{\mu}^{3 / 2}=\ell \frac{2}{3 \sqrt{3}} \mu^{3 / 2} .
$$

Setting $\mu=\lambda\left(8 \pi G_{4} / 3\right) \hat{\rho}$ and $\ell^{-2}=\left(4 \pi G_{4} / 3\right) \sigma$, we get

$$
q^{4}<\frac{4}{27}\left(\frac{3}{4 \pi G_{4} \sigma}\right) \lambda^{3}\left(\frac{8 \pi G_{4} \hat{\rho}}{3}\right)^{3} .
$$

The two constraints are compatible if

$$
\lambda^{3}-\frac{27}{4}(1+\lambda)=\left(\lambda+\frac{3}{2}\right)^{2}(\lambda-3)>0 .
$$

This inequality holds only for $\lambda>3$ and cannot be satisfied for values as low as $\lambda \sim 0.14$, which follows from the nucleosynthesis constraint. Thus, unfortunately, the charge value required for the occurrence of the bounce corresponds to an extremal black hole background.

An alternative to the two-horizon constraint, which also puts an upper bound on the value of the charge parameter, can be obtained from the requirement that the energy density of the universe at the bouncing point must be larger than the one at the time of nucleosynthesis, i.e. $\hat{\rho} / R_{\text {min }}^{4}$ $>(0.2 \mathrm{MeV})^{4}$. This constraint was mentioned in [17] but was not properly addressed as the authors lacked the exact solution for the scale factor. The value of $R_{\min }$ varies as a function of the parameters $q^{2}, \mu$ and $\hat{\rho}$ according to Eq. (28). The strongest constraint arises by considering the maximal possible value of $R_{\min }$, and thus the minimal possible value of $\rho$, which corresponds to large values of $q^{2}$ and is given by $R_{\min }^{2} \simeq q^{2} / b$. Substituting this value in the expression of the energy density, we obtain the constraint

$$
q^{4}<\frac{(1+\lambda)^{2} \hat{\rho}}{(0.2 \mathrm{MeV})^{4}}\left(\frac{8 \pi G_{4}}{3} \hat{\rho}\right)^{2}
$$

The above upper bound on the value of charge parameter replaces Eq. (33) and is necessary for the validity of the bouncing solution in an extremal black hole, fivedimensional background. The requirement, finally, that the quadratic energy-density term be subdominant compared to the linear one, at the time of nucleosynthesis, leads to

$$
\sigma>\frac{1}{2} \frac{\hat{\rho}}{R^{4}} \simeq(0.17 \mathrm{MeV})^{4}
$$

a value which is smaller than the one derived in Ref. [17].

\section{MATTER DOMINATED BRANE}

Concluding our study of the evolution of a fourdimensional brane embedded in a symmetric, AdS charged black-hole, bulk spacetime, we will now study the case of a matter equation of state for the energy density on the brane. In that case, we have $\rho=\tilde{\rho} / R^{3}$ and the Friedmann equation (15) takes the form

$$
\begin{aligned}
H^{2}= & \left(\frac{\dot{R}}{R}\right)^{2} \\
= & \frac{8 \pi G_{4}}{3} \Lambda_{4}-\frac{k}{R^{2}}+\frac{\mu}{R^{4}}+\frac{8 \pi G_{4}}{3} \frac{\tilde{\rho}}{R^{3}} \\
& +\left(\frac{4 \pi G_{4}}{3 \sigma} \tilde{\rho}^{2}-q^{2}\right) \frac{1}{R^{6}} .
\end{aligned}
$$

Given the relevance of this particular equation of state at late times in the history of the universe, it would not be meaningful to talk about the existence or not of an initial singularity. For large values of $R$, the charge as well as the quadratic energy-density term is subdominant and can be safely dropped. The $\mu$ term remains and the relevant question is how this term, remnant of the structure of the 5-dimensional 
TABLE III. Neutral $\left(Q^{2}=0\right)$, critical $\left(\Lambda_{4}=0\right)$, matter-dominated brane.

\begin{tabular}{llr}
\hline \hline$k=1$ & $R=\frac{A}{2}\left\{1+\sqrt{\left.1+\frac{4 \mu}{A^{2}} \sin \left[\eta-\eta_{0}+\arctan \left(\frac{2 R_{0}-A}{2 \sqrt{\mu+A R_{0}-R_{0}^{2}}}\right)\right]\right\}} \begin{array}{c}\text { Expanding- } \\
\text { contracting }\end{array}\right.$ \\
\hline$k=0$ & $R=R_{0}+\frac{A}{4}\left(\eta-\eta_{0}\right)^{2}+\sqrt{\mu+A R_{0}}\left(\eta-\eta_{0}\right)$ & $\begin{array}{c}\text { Power-law } \\
\text { expanding }\end{array}$ \\
$k=-1$ & $R=\frac{A}{2}\left\{-1+\sqrt{1-\frac{4 \mu}{A^{2}}} \cosh \left[\eta-\eta_{0}-\ln \left(\frac{\sqrt{A^{2}-4 \mu}}{2 \sqrt{\mu+A R_{0}+R_{0}^{2}}+A+2 R_{0}}\right)\right]\right\}$ & $\begin{array}{r}\text { Exponentially } \\
\text { expanding }\end{array}$ \\
\hline \hline
\end{tabular}

bulk, affects the evolution of the brane at the late time regime. For simplicity, we will consider again a critical brane with $\Lambda_{4}=0$ and solve for the scale factor, for $k=0, \pm 1$. By using the conformal time coordinate $d \tau=R(\eta) d \eta$, we obtain the solutions listed in Table III.

In the above, we have defined $A=\left(8 \pi G_{4} / 3\right) \tilde{\rho}$ and have denoted with $R_{0}$ the value of the scale factor at the beginning of the matter-dominated era, at $\eta=\eta_{0}$.

For $k=1$, the matter-dominated brane first expands and then contracts, in agreement with the standard cosmological model. At the point where $H$, or equivalently $d R / d \eta$, becomes zero, the universe stops expanding and then recollapses. This occurs at

$$
R=\frac{A}{2}\left(1+\sqrt{1+\frac{4 \mu}{A^{2}}}\right),
$$

and it clearly corresponds to a larger value of the scale factor compared to the case where $\mu=0$. The main implication, therefore, of the bulk parameters on the evolution of the closed, matter-dominated brane, at large scales, is the increase of the proper volume of the universe. As the brane contracts, we will eventually reach small values of the scale factor for which the charge term will become dominant again. In that case, the evolution of the brane would be governed by the equation

$$
R^{4} \dot{R}^{2}=\mu R^{2}-\tilde{q}^{2},
$$

where we have defined $\widetilde{q}^{2}=q^{2}-\left(4 \pi G_{4} / 3 \sigma\right) \tilde{\rho}^{2}$ and ignored the curvature and linear energy-density terms which are now subdominant. Clearly, the above equation is characterized by the vanishing of $\dot{R}$ at a finite value of the scale factor, namely at $R_{\text {min }}^{2}=\tilde{q}^{2} / \mu$, as long as $\tilde{q}^{2}>0$, a constraint that puts a lower bound on the charge parameter. If the constraint for the existence of two horizons (33) had not been violated in the precedented radiation-dominated era, one could have shown that the two constraints on $q^{2}$ would have been indeed compatible, in the matter-dominated era, if $\sigma$ $>\left(4 \pi G_{4}\right)^{3} \widetilde{\rho}^{4} / 4 \mu^{3}$. If the alternative upper bound (35) is used instead, we derive the constraint

$$
\sigma>\frac{(0.2 \mathrm{MeV})^{2}}{2(1+\lambda)} \frac{\tilde{\rho}^{2}}{\hat{\rho}^{3 / 2}} .
$$

For $k=0$ and -1 , the four-dimensional brane expands forever and no future singularity is encountered, as expected. In the case of a flat universe, the $\mu$ term adds a positive contribution to the value of the scale factor and thus increases the rate of expansion. For an open universe, however, and for a given time $\eta$, we may easily see that the value of the scale factor is smaller compared to the one for $\mu=0$, and therefore the bulk parameter delays the expansion of the universe in this case. The derived solution is valid as long as

$$
\omega_{4} M<\frac{1}{4}\left(\frac{8 \pi G_{4}}{3} \tilde{\rho}\right)^{2},
$$

which puts an upper bound on the black hole mass $M$.

\section{BOUNCING IN AN ASYMMETRIC BACKGROUND}

In this section, we shall consider the possibility of an asymmetric bulk space consisting of two distinct regions terminating on the brane. To keep things simple, we shall consider for both regions a charged AdS black hole geometry characterized by the same AdS length $\ell$ and charge $|Q|$ but with different black hole masses $M_{ \pm}$. We shall denote the two metric functions as

$$
f_{ \pm}(R)=\frac{R^{2}}{\ell^{2}}+k-\frac{\mu_{ \pm}}{R^{2}}+\frac{q^{2}}{R^{4}}
$$

The Friedmann equation takes the form (13) which can be squared twice to give

$$
\dot{R}^{2}+k=\frac{\mu}{R^{2}}-\frac{q^{2}}{R^{4}}+\frac{2 R^{2}}{\ell^{2}}\left(\frac{\rho}{\sigma}\right)\left(1+\frac{\rho}{2 \sigma}\right)+\frac{\ell^{2}}{16 R^{6}} \frac{(\Delta \mu)^{2}}{\left(1+\frac{\rho}{\sigma}\right)^{2}},
$$

where we have assumed a critical brane by making the same fine-tuning as in the symmetric case, namely

$$
\left(\frac{4 \pi G \sigma}{3}\right)^{2}=\frac{1}{\ell^{2}}=\frac{4 \pi G_{4}}{3} \sigma,
$$

and have also defined

$$
\mu \equiv \frac{1}{2}\left(\mu_{+}+\mu_{-}\right), \quad(\Delta \mu)^{2} \equiv\left(\mu_{+}-\mu_{-}\right)^{2} .
$$


It is straightforward to see that, in the case $\mu_{+}=\mu_{-}$, the Friedmann equation (15) for a symmetric bulk is recovered.

In the case of an empty, critical brane $(\rho=0)$ the above evolution equation simplifies to

$$
\left(\frac{\dot{R}}{R}\right)^{2}+\frac{k}{R^{2}}=\frac{\mu}{R^{4}}-\frac{q^{2}}{R^{6}}+\frac{\ell^{2}(\Delta \mu)^{2}}{16 R^{8}} .
$$

As a result of the asymmetry, there is a positive term present that opposes the effects of the charge at small values of the scale factor. In the same early regime, the curvature term can be dropped. Then, the above equation has exactly the same form as the Friedmann equation in the case of a critical, radiation-dominated universe with a symmetric bulk, with $(\Delta \mu)^{2}$ playing the role of the quadratic energy-density term, and thus possesses a bouncing solution for large enough charge. Introducing again $x=R^{2}$ and $d \tau=R^{2} d \bar{\tau}$, we can bring Eq. (46) in the form

$$
\frac{1}{4}\left(x^{\prime}\right)^{2}=\mu x^{2}-q^{2} x+\frac{\ell^{2}(\Delta \mu)^{2}}{16}
$$

from which we obtain the solution

$$
R^{2}=\frac{q^{2}}{2 \mu}\left\{1+\sqrt{1-\frac{\mu \ell^{2}(\Delta \mu)^{2}}{4 q^{4}}} \cosh (2 \sqrt{\mu} \bar{\tau})\right\},
$$

for large enough values of the charge, namely

$$
q^{4}>\frac{\mu \ell^{2}(\Delta \mu)^{2}}{4} \text {. }
$$

This solution is characterized by a minimum value of the scale factor

$$
R_{\min }^{2}=\frac{q^{2}}{2 \mu}\left\{1+\sqrt{1-\frac{\mu \ell^{2}(\Delta \mu)^{2}}{4 q^{4}}}\right\}
$$

obtained $^{6}$ at $\bar{\tau}=\tau=0$ where the bouncing occurs.

The above lower limit on the charge should be compared with the upper limit required by the non-extremality of the background $^{7}$ namely,

$$
\bar{q}^{2}<\bar{q}_{+}^{2}=\frac{2}{3 \sqrt{3}} \min \left\{\bar{\mu}_{ \pm}^{3}\right\}
$$

We are, thus, eventually led to the condition

$$
\begin{aligned}
& { }^{6} \text { The two times are related through } \\
& \qquad \tau=\frac{q^{2}}{4 \mu^{3 / 2}}\left[2 \sqrt{\mu} \bar{\tau}+\left(1-\frac{\mu \ell^{2}(\Delta \mu)^{2}}{4 q^{4}}\right)^{1 / 2} \sinh (2 \sqrt{\mu} \bar{\tau})\right] .
\end{aligned}
$$

\footnotetext{
${ }^{7}$ Since the curvature term is always subdominant in the regime where the bounce occurs, for simplicity we consider the $k=0$ condition.
}

$$
\left(\mu_{+}-\mu_{-}\right)^{2}<\frac{32}{27} \frac{\min \left\{\mu_{ \pm}^{3}\right\}}{\left(\mu_{+}+\mu_{-}\right)} .
$$

Therefore, a bounce occurs with the black hole background possessing two distinct horizons provided the asymmetry is not too large.

What about a radiation-dominated brane? In that case, the Friedmann equation is of the form

$$
\begin{aligned}
\dot{R}^{2}+k= & \frac{1}{R^{2}}\left[\mu+\frac{2}{\ell^{2}}\left(\frac{\hat{\rho}}{\sigma}\right)\right]-\frac{q^{2}}{R^{4}}+\frac{1}{\ell^{2}}\left(\frac{\hat{\rho}}{\sigma}\right)^{2} \frac{1}{R^{6}} \\
& +\frac{\ell^{2}(\Delta \mu)^{2}}{16\left(\frac{\hat{\rho}}{\sigma}\right)^{2}} \frac{R^{2}}{\left[1+\left(\frac{\sigma}{\hat{\rho}}\right) R^{4}\right]^{2}},
\end{aligned}
$$

where we have introduced $\rho=\hat{\rho} R^{-4}$. For small values of the scale factor, we can approximate this equation with

$$
\dot{R}^{2} \simeq \frac{1}{R^{2}}\left[\mu+\frac{2}{\ell^{2}}\left(\frac{\hat{\rho}}{\sigma}\right)\right]-\frac{q^{2}}{R^{4}}+\frac{1}{\ell^{2}}\left(\frac{\hat{\rho}}{\sigma}\right)^{2} \frac{1}{R^{6}} .
$$

Note that the asymmetry, in contrast to the empty-brane case, contributes only with a sub-leading term $\left[\ell^{2}(\Delta \mu)^{2} \sigma^{2} / 16 \hat{\rho}^{2}\right] R^{2}$, which can be dropped to a first approximation in our considerations concerning the occurrence of a bounce. The remaining equation is identical to the one in the symmetric case and yields essentially the same lethal condition

$$
1+\frac{1}{2}\left(\lambda_{+}+\lambda_{-}\right)<\frac{4}{27} \min \left\{\lambda_{ \pm}^{3}\right\}
$$

with the $\lambda$ 's being defined as $\mu_{ \pm}=\lambda_{ \pm}\left(8 \pi G_{4} \hat{\rho} / 3\right)$. The quantity $\left(\lambda_{+}+\lambda_{-}\right) / 2$ is still constrained by nucleosynthesis to be smaller than 0.14 , a result which is in contradiction with the above inequality: setting $\left(\lambda_{+}+\lambda_{-}\right) / 2 \simeq 0.13$, we are led to the constraint $\min \left\{\lambda_{ \pm}\right\}>1.9$, which cannot be true given the constraint on their sum and the positivedefiniteness of $\lambda_{ \pm}$.

In the case, finally, of a matter-dominated universe with $\rho=\tilde{\rho} / R^{3}$, the Friedmann equation (43) becomes

$$
\dot{R}^{2}+k=\frac{\mu}{R^{2}}+\left(\frac{\tilde{\rho}^{2}}{\ell^{2} \sigma^{2}}-q^{2}\right) \frac{1}{R^{4}}+\frac{2 \tilde{\rho}}{\ell^{2} \sigma} \frac{1}{R}+\frac{\ell^{2}(\Delta \mu)^{2}}{16\left(R^{3}+\tilde{\rho} / \sigma\right)^{2}} .
$$

At large scales, the $(\Delta \mu)^{2}$ term has an $R^{-6}$ dependence which makes this term negligible compared to the remaining ones. In the same way, at small scales, this term has the same scaling as the curvature term and is again subdominant. Therefore, an asymmetric bulk has no effect on the evolution of a matter-dominated universe. 


\section{EFFECTS OF AN INTRINSIC CURVATURE TERM}

It has been pointed out [36] that the divergence arising for the energy-momentum tensor at the boundary of the Schwarzschild-AdS space requires the introduction of an intrinsic curvature scalar counterterm. Such a term, arising in other frameworks as well [37], is certainly not forbidden. We shall, thus, assume the presence in the action of the term [38]

$$
\Delta \mathcal{S}=\frac{\beta \ell}{32 \pi G} \int d^{4} x \sqrt{-\gamma} \mathcal{R}_{4},
$$

where $\beta$ is a dimensionless constant that controls the "turning on" and "off" of the boundary curvature term. A priori, such an addition is mostly expected to modify the "late," or large-scale, evolution of the brane and not the small-scale behavior responsible for the bounce. The resulting evolution equation on the brane, for a $\mathcal{Z}_{2}$-symmetric bulk spacetime, is

$$
2 \sqrt{\dot{R}^{2}+f(R)}=\frac{8 \pi G}{3} R(\rho+\sigma)-\frac{\beta \ell}{2 R}\left(\dot{R}^{2}+k\right),
$$

with our standard metric function $f(R)=k-\mu / R^{2}+q^{2} / R^{4}$ $+R^{2} / \ell^{2}$. Taking the square of the above equation, we obtain a quadratic algebraic equation with solution

$$
\dot{R}^{2}+k=\frac{8 R^{2}}{(\beta \ell)^{2}}\left\{1+\frac{\beta \ell}{2}\left(\frac{4 \pi G}{3}\right)(\rho+\sigma)-\sqrt{1+\frac{\beta^{2}}{4}+\beta \ell\left(\frac{4 \pi G}{3}\right)(\rho+\sigma)-\frac{(\beta \ell)^{2}}{4}\left(\frac{\mu}{R^{4}}-\frac{q^{2}}{R^{6}}\right)}\right\} .
$$

In what follows, we will perform the same fine-tuning that, in the case $\rho=0$ and $\beta=0$, leads to a critical brane, namely $\ell^{-1}=(4 \pi G / 3) \sigma$.

Considering first the case of an empty brane $(\rho=0)$, we obtain the equation

$$
\dot{R}^{2}+k=\frac{4(\beta+2)}{(\beta \ell)^{2}} R^{2}\left\{1-\sqrt{1-\left(\frac{\beta \ell}{\beta+2}\right)^{2}\left(\frac{\mu}{R^{4}}-\frac{q^{2}}{R^{6}}\right)}\right\} .
$$

For small values of the parameter $\beta$, this equation can be replaced with

$$
\dot{R}^{2}+k \simeq \frac{2 R^{2}}{(\beta+2)}\left(\frac{\mu}{R^{4}}-\frac{q^{2}}{R^{6}}\right),
$$

which has the solutions displayed in Table I of Sec. IV, with the parameter rescaling

$$
\begin{aligned}
& \mu \rightarrow \mu /\left(1+\frac{\beta}{2}\right), \quad q^{2} \rightarrow q^{2} /\left(1+\frac{\beta}{2}\right), \\
& \epsilon^{2} \rightarrow\left(1+\frac{\beta}{2}\right) \epsilon^{2} .
\end{aligned}
$$

The smallness of $\beta$ required for the validity of the above approximation is $\beta^{2} \ll \mu^{3} / \ell^{2} q^{4} \sim q_{+}^{2} / q^{2}<1$.

For an appreciable value of $\beta$, such as the counterterm value $\beta=1$, the above equation cannot be integrated analytically but the expectation that the small-scale behavior is not going to be modified can be clarified by some supportive arguments. Ignoring the curvature term proportional to $k$, we can rewrite the expression under the square-root symbol in a manifestly positive fashion in terms of the metric function $f(R)$, which is positive for all points outside the outer horizon. Our equation is

$$
H^{2}=\frac{4(\beta+2)}{(\beta \ell)^{2}}\left\{1-\sqrt{\frac{4(\beta+1)}{(\beta+2)^{2}}+\frac{f(R)}{R^{2}}\left(\frac{\beta \ell}{\beta+2}\right)^{2}}\right\} .
$$

Positivity of $H^{2}$ demands

$$
1>\frac{4(\beta+1)}{(\beta+2)^{2}}+\frac{f(R)}{R^{2}}\left(\frac{\beta \ell}{\beta+2}\right)^{2},
$$

which turns out to be $\beta$ independent, namely

$$
\frac{f(R)}{R^{2}}<\frac{1}{\ell^{2}} \Rightarrow R^{2}>\frac{q^{2}}{\mu} \equiv R_{\text {min }}^{2} .
$$

Thus, the minimal value of the scale factor, obtained in this way, turns out to be $\beta$-independent.

In the case of non-zero energy density on the brane ( $\rho$ $\neq 0$ ), our Friedmann equation is

$$
\begin{aligned}
H^{2}+\frac{k}{R^{2}}= & \frac{8}{(\beta \ell)^{2}}\left\{1+\frac{\beta}{2}\left(1+\frac{\rho}{\sigma}\right)\right. \\
& -\sqrt{\left(1+\frac{\beta}{2}\right)^{2}+\beta\left(\frac{\rho}{\sigma}\right)-\frac{(\beta \ell)^{2}}{4}\left(\frac{\mu}{R^{4}}-\frac{q^{2}}{R^{6}}\right)} .
\end{aligned}
$$

Ignoring the $k$ term, we can repeat the argument we used in the $\rho=0$ case and arrive again at a $\beta$-independent condition

$$
\frac{\mu}{R^{4}}-\frac{q^{2}}{R^{6}}+\frac{1}{\ell^{2}}\left[2 \frac{\rho}{\sigma}+\left(\frac{\rho}{\sigma}\right)^{2}\right]>0,
$$


which, for example, for $\rho$ corresponding to radiation ( $\rho$ $\left.=\hat{\rho} R^{-4}\right)$ leads to our well-known constraint $q^{4}$ $>4(\hat{\rho} / \sigma \ell)^{2}(\mu+2 \hat{\rho} / \sigma \ell)$.

At large scales, the curvature is, of course, expected to influence the evolution. For large $R$, but not necessarily small $\beta$, we derive again Eq. (61) for an empty brane. The solutions are again obtained from Table I of Sec. IV by using the rescaled parameters (62). For the cases $k=0,-1$, for which $R$ is eternally expanding after the bounce, there is always a value of $R$ large enough for the approximation to be trusted for any value of $\beta$. In the cyclic universe, however, obtained for $k=1$, there is a maximum value of the scale factor given by $R_{\max }^{2}=\mu /(\beta+2)$. When substituted in the expression under the square root in the exact equation (60), with the charge term having been neglected as subdominant, a term $(\beta \ell)^{2} / \mu$ arises, which needs to be small compared to unity for our approximation to be valid. Even for values of $\beta$ of $\mathcal{O}(1)$, this term is indeed negligible provided that the black hole mass-length parameter $\sqrt{\mu}$ is much larger than the AdS length $\ell$. In that case, the cyclic behavior of the $k=1$ solution is retained for any value of $\beta$; in the opposite case, only small values of $\beta$ are allowed.

\section{CONCLUSIONS AND DISCUSSION}

As in the case of an AdS bulk spacetime, the generalized Friedmann equation derived on a brane embedded in an AdS-black-hole bulk spacetime allows for modifications in the evolution of the four-dimensional subspace at small scales. This result allows us to study the early time regime, as well as the late-time regime for closed universes, and investigate whether the corresponding cosmological singularities can be indeed avoided. The main attractive feature of the brane-world model considered in the present article, in which the five-dimensional spacetime is described by an AdS charged black hole, is the fact that it realizes the bounce idea: the existence of a non-zero minimum value of the scale factor that smoothly connects a contracting with an expanding phase in the evolution of the four-dimensional subspace. In all cases considered, this is indeed possible for a nonvanishing value of the charge parameter of the fivedimensional black hole. The bounce effect therefore predicted in the charged AdS black hole background provides support for a singularity-free cosmology in which the big bang singularity is not present, as well as for a cyclic universe [39] scenario in which neither the Big Bang nor a big crunch singularity is present.

Unfortunately, it is not possible to formulate a model that would allow us to study both the early and late time regimes in the history of the universe, since different epochs are dominated by different energy densities. It is therefore necessary to distinguish between regions with smoothly connected but differing equations of state, an approach followed here in chronological order. By studying first, in Sec. IV, the case of an empty brane with either a zero or non-zero cosmological constant, embedded in a charged AdS-black-hole bulk spacetime, and joining together the two sets of solutions, we were able to model a singularity-free, early infla- tionary era: the solutions are free from the big bang singularity and they smoothly interpolate to a de Sitter expanding phase. The derived constraints on the various parameters of the model put an upper bound on the Hubble parameter of the asymptotic de Sitter phase which, when combined with the demand that the magnitude of the density perturbations produced in this period have the correct size, leads to an intermediate-scale higher-dimensional gravitational theory, i.e. $M_{5}>10^{-5} M_{P}$.

We might assume instead that the early time regime is dominated by a radiation-type equation of state. The derivation of the exact solution for the scale factor on the brane, at small scales, confirms the existence of a bouncing and the absence of the big bang singularity. Assuming that this singularity-free, radiation-dominated epoch lasts until the time of nucleosynthesis without interruption, we are forced to satisfy a stringent constraint on the maximum value of the radiation-type energy-density term that appears in the Friedmann equation. As our analysis revealed, the range of parameters of the background, for which the bouncing is possible and the nucleosynthesis constraint is satisfied, exceeds the limit allowed by a non-extremal black hole and may lead to an unstable background. This problem may be avoided by assuming that the dominant equation of state does not remain the same for the whole range of values from the bouncing point to the time of nucleosynthesis. Since the universe must be radiation dominated at nucleosynthesis time, that leaves two options: (i) either the equation of state is dominated, at the bouncing point, by the cosmological constant, which then leads to an inflationary period and finally to a late radiationdominated period, or (ii) an early radiation-type equation of state gives way to an intermediate inflationary period, as mentioned in Sec. V, before coming back to a late radiationdominated period at the time of nucleosynthesis.

As the universe expands, the radiation-dominated energy density becomes subdominant and gives its place to the matter-dominated one. In Sec. VI, we studied the modifications that the generalized Friedmann equation brings to the evolution of the brane at this large-scale regime. In the case of an open or flat brane, the charge-dependent term is always negligible and it is only the black-hole-mass-dependent term that survives and affects the expansion rate of the brane while preserving the eternal expansion predicted by the fourdimensional cosmological model. In the case of a closed brane, the latter term causes an increase in the proper volume of the universe but it cannot prevent subsequent collapse. Assuming that the equation of state remains matter dominated during this late small-scale regime, the charge term becomes dominant and ensures passage from the contracting to a subsequent expanding phase and, thus, avoidance of the big crunch.

However, cosmological observations [40-43] strongly indicate that the present universe is spatially flat and accelerating due to some dominant dark-energy component. The simplest possibility is that this dark energy of unknown origin is in the form of a small cosmological constant that puts the universe in an indefinitely expanding de Sitter phase. This scenario can be easily accommodated in the framework of the second set of solutions derived in Sec. IV, which pre- 
dict an asymptotic de Sitter expansion for all values of $k$. The same solutions could also model the alternative scenario in which the dark energy is generated by a slowly varying scalar field [44], with a $w \simeq-1$ equation of state and thus an almost constant energy density. In such a scenario, the derived de Sitter expanding phase is only an intermediate one that eventually will give way to an asymptotic Minkowski regime as the speed of expansion will start decreasing. In both cases, it is only the black-hole mass parameter that is relevant to the present-time evolution, by restricting the Hubble parameter for the, either eternal or temporary, de Sitter expansion phase, while the charge parameter has absolutely no effect.

In addition to the uncertainty about the presently valid equation of state, the very late evolution is also open to speculation and conjecture, leaving open the possibility of a contracting and, perhaps, cyclic, behavior. If, for example, the dark energy eventually becomes negative, the universe will collapse [45]. In the cosmic contraction scenario, the background charge will be essential in avoiding a big crunch and bouncing back into an expanding state, just like in the case of a matter-dominated phase. Nevertheless, the specific energy density required for a late-time contracting phase has to be inserted in the brane energy-momentum tensor in an arbitrary fashion. The fundamental physics associated with its required form is still lacking. An interesting and perhaps fruitful approach would be to try to investigate ways of nontrivial bulk-brane interactions resulting, through the ex- change of energy [46], in a dynamical evolution of the equation of state on the brane that accounts for the present time accelerating phase as well as for a possible contracting one.

Let us finally note that, according to our analysis conducted in Secs. VII and VIII, variants of the above model, in which the bulk spacetime is assumed to be asymmetric or a brane curvature term is added in the action, do not lead to any radical changes in the type of behavior encountered near, or the existence itself of, the bouncing point. In the first variant, it is only in the case of an empty brane and, for large values of the black-hole mass difference on the two sides of the brane, that the extra term in the Friedmann equation tends to prohibit the occurrence of the bouncing. In every other case, the effect of this term is irrelevant. In the second variant, the brane curvature term has an effect only at large scales, as expected, and can be ignored at the time of the bouncing, either at early or late times, without any loss of information.

\section{ACKNOWLEDGMENTS}

P.K. would like to thank the Theoretical Physics Group at the Technical University of Munich, where parts of this work were completed, for its hospitality and financial support. K.T. would like to thank the CERN Theory Division for its hospitality and A. Petkou for useful discussions. He also acknowledges traveling support from the RTN program HPRNCT-2000-00152.
[1] V.A. Rubakov and M.E. Shaposhnikov, Phys. Lett. 125B, 136 (1983); 125B, 139 (1983); K. Akama, Lect. Notes Phys. 176, 267 (1982).

[2] J. Polchinski, Phys. Rev. Lett. 75, 4724 (1995); hep-th/9611050.

[3] P. Horava and E. Witten, Nucl. Phys. B460, 506 (1996).

[4] J.M. Maldacena, Adv. Theor. Math. Phys. 2, 231 (1998); S.S. Gubser, I.R. Klebanov, and A.M. Polyakov, Phys. Lett. B 428, 105 (1998); E. Witten, Adv. Theor. Math. Phys. 2, 253 (1998).

[5] I. Antoniadis, Phys. Lett. B 246, 377 (1990).

[6] I. Antoniadis, S. Dimopoulos, and G.R. Dvali, Nucl. Phys. B516, 70 (1998).

[7] I. Antoniadis, N. Arkani-Hamed, S. Dimopoulos, and G.R. Dvali, Phys. Lett. B 436, 257 (1998).

[8] L. Randall and R. Sundrum, Phys. Rev. Lett. 83, 3370 (1999).

[9] L. Randall and R. Sundrum, Phys. Rev. Lett. 83, 4690 (1999).

[10] M. Gogberashvili, Mod. Phys. Lett. A 14, 2025 (1999).

[11] P. Binetruy, C. Deffayet, and D. Langlois, Nucl. Phys. B565, 269 (2000); N. Kaloper, Phys. Rev. D 60, 123506 (1999); T. Nihei, Phys. Lett. B 465, 81 (1999); C. Csaki, M. Graesser, C.F. Kolda, and J. Terning, ibid. 462, 34 (1999); J.M. Cline, C. Grojean, and G. Servant, Phys. Rev. Lett. 83, 4245 (1999); T. Shiromizu, K.i. Maeda, and M. Sasaki, Phys. Rev. D 62, 024012 (2000); P. Kanti, I.I. Kogan, K.A. Olive, and M. Pospelov, Phys. Lett. B 468, 31 (1999); Phys. Rev. D 61, 106004 (2000); P. Binetruy, C. Deffayet, U. Ellwanger, and D. Langlois, Phys. Lett. B 477, 285 (2000); E.E. Flanagan, S.H.
Tye, and I. Wasserman, Phys. Rev. D 62, 044039 (2000); C. Csaki, M. Graesser, L. Randall, and J. Terning, ibid. 62, 045015 (2000); R. Maartens, D. Wands, B.A. Bassett, and I. Heard, ibid. 62, 041301(R) (2000); P. Kanti, K.A. Olive, and M. Pospelov, Phys. Lett. B 481, 386 (2000); Phys. Rev. D 62, 126004 (2000); N. Deruelle and T. Dolezel, ibid. 62, 103502 (2000); A. Kehagias and K. Tamvakis, Phys. Lett. B 515, 155 (2001).

[12] P. Kraus, J. High Energy Phys. 12, 011 (1999); C. Barcelo and M. Visser, Phys. Lett. B 482, 183 (2000).

[13] A. Kehagias and E. Kiritsis, J. High Energy Phys. 11, 022 (1999).

[14] A.C. Petkou and G. Siopsis, J. High Energy Phys. 02, 045 (2002).

[15] A.J. Medved, hep-th/0205037.

[16] R.B. Mann, Nucl. Phys. B516, 357 (1998).

[17] S. Mukherji and M. Peloso, Phys. Lett. B 547, 297 (2002).

[18] A.J. Medved, hep-th/0205251.

[19] D.H. Coule, Class. Quantum Grav. 18, 4265 (2001); gr-qc/0202104.

[20] J.P. Gregory and A. Padilla, Class. Quantum Grav. 19, 4071 (2002); A. Padilla, hep-th/0210217.

[21] S. Nojiri, S.D. Odintsov, and S. Ogushi, Int. J. Mod. Phys. A 17, 4809 (2002).

[22] S. Kachru and L. McAllister, J. High Energy Phys. 03, 018 (2003).

[23] C.P. Burgess, F. Quevedo, S.J. Rey, G. Tasinato, and I. Zavala, 
J. High Energy Phys. 10, 028 (2002).

[24] P. Brax and D.A. Steer, Phys. Rev. D 66, 061501(R) (2002).

[25] Y. Shtanov and V. Sahni, Phys. Lett. B 557, 1 (2003).

[26] P. Singh and N. Dadhich, hep-th/0208080.

[27] Y.S. Myung, Class. Quantum Grav. 20, 935 (2003).

[28] A. Biswas, S. Mukherji, and S.S. Pal, hep-th/0301144.

[29] R.H. Brandenberger and C. Vafa, Nucl. Phys. B316, 391 (1989); R.H. Brandenberger, V. Mukhanov, and A. Sornborger, Phys. Rev. D 48, 1629 (1993); I. Antoniadis, J. Rizos, and K. Tamvakis, Nucl. Phys. B415, 497 (1994); J. Rizos and K. Tamvakis, Phys. Lett. B 326, 57 (1994); N. Kaloper, R. Madden, and K.A. Olive, Nucl. Phys. B452, 677 (1995); R. Easther and K.i. Maeda, Phys. Rev. D 54, 7252 (1996); S. Kalyana Rama, Phys. Rev. Lett. 78, 1620 (1997); M. Gasperini, M. Maggiore, and G. Veneziano, Nucl. Phys. B494, 315 (1997); P. Kanti, J. Rizos, and K. Tamvakis, Phys. Rev. D 59, 083512 (1999); R.H. Brandenberger, R. Easther, and J. Maia, J. High Energy Phys. 08, 007 (1998).

[30] R.G. Cai, Phys. Rev. D 63, 124018 (2001).

[31] A. Chamblin, R. Emparan, C.V. Johnson, and R.C. Myers, Phys. Rev. D 60, 104026 (1999).

[32] N. Kaloper and A.D. Linde, Phys. Rev. D 59, 101303 (1999); P. Kanti and K.A. Olive, ibid. 60, 043502 (1999); Phys. Lett. B 464, 192 (1999); N. Kaloper and A.R. Liddle, Phys. Rev. D 61, 123513 (2000); M. Bastero-Gil, V. Di Clemente, and S.F. King, ibid. 67, 083504 (2003).

[33] E. Lisi, S. Sarkar, and F.L. Villante, Phys. Rev. D 59, 123520 (1999).

[34] K.A. Olive, G. Steigman, and T.P. Walker, Phys. Rep. 333, 389 (2000).

[35] S.H. Hansen, G. Mangano, A. Melchiorri, G. Miele, and O. Pisanti, Phys. Rev. D 65, 023511 (2002).

[36] V. Balasubramanian and P. Kraus, Commun. Math. Phys. 208, 413 (1999); P. Kraus, F. Larsen, and R. Siebelink, Nucl. Phys. B563, 259 (1999).
[37] G.R. Dvali, G. Gabadadze, and M. Porrati, Phys. Lett. B 485, 208 (2000); C. Deffayet, ibid. 502, 199 (2001); C. Deffayet, G.R. Dvali, and G. Gabadadze, Phys. Rev. D 65, 044023 (2002).

[38] H. Collins and B. Holdom, Phys. Rev. D 62, 105009 (2000); Y.V. Shtanov, hep-th/0005193; N.J. Kim, H.W. Lee, and Y.S. Myung, Phys. Lett. B 504, 323 (2001).

[39] P.J. Steinhardt and N. Turok, astro-ph/0204479; Science 296, 1436 (2002).

[40] Supernova Search Team Collaboration, A.G. Riess et al., Astron. J. 116, 1009 (1998); Supernova Cosmology Project Collaboration, S. Perlmutter et al., ibid. 517, 565 (1999).

[41] J.L. Sievers et al., astro-ph/0205387.

[42] D. Pogosyan, J.R. Bond, and C.R. Contaldi, astro-ph/0301310.

[43] P.J. Peebles and B. Ratra, Rev. Mod. Phys. 75, 559 (2003).

[44] A.D. Dolgov, in The Very Early Universe, edited by G.W. Gibbons, S.W. Hawking, and S. Siklos (Cambridge University Press, Cambridge, England, 1983), pp. 449-458; C. Wetterich, Nucl. Phys. B302, 668 (1988); B. Ratra and P.J. Peebles, Phys. Rev. D 37, 3406 (1988); P.G. Ferreira and M. Joyce, ibid. 58, 023503 (1998); I. Zlatev, L.M. Wang, and P.J. Steinhardt, Phys. Rev. Lett. 82, 896 (1999).

[45] R. Kallosh and A. Linde, J. Cosmol. Astropart. Phys. 02, 002 (2003); A.D. Linde, in Three Hundred Years of Gravitation, edited by S.W. Hawking and W. Israel (Cambridge University Press, Cambridge, England, 1987), pp. 604-630; L.M. Krauss and M.S. Turner, Gen. Relativ. Gravit. 31, 1453 (1999); G.N. Felder, A.V. Frolov, L. Kofman, and A.V. Linde, Phys. Rev. D 66, 023507 (2002).

[46] E. Kiritsis, G. Kofinas, N. Tetradis, T.N. Tomaras, and V. Zarikas, J. High Energy Phys. 02, 035 (2003); A. Hebecker and J. March-Russell, Nucl. Phys. B608, 375 (2001); D. Langlois, L. Sorbo, and M. Rodriguez-Martinez, Phys. Rev. Lett. 89, 171301 (2002); N. Tetradis, hep-th/0211200. 